\title{
Electronic Markets on academic supply chains
}

\author{
Rainer Alt ${ }^{1}$
}

Published online: 3 May 2017

(C) Institute of Applied Informatics at University of Leipzig 2017

Dear readers of Electronic Markets,

This issue represents a double special issue consisting of papers from the special issue on "Electronic Markets in supply chain management" and the special issue on the "Transformation of the academic publishing market". Although, the calls for both special issues co-evolved without initially being foreseen for publication in a joint issue, both topics have a close link to Electronic Markets as well as to each other. In the history of Electronic Markets, a number of issues have appeared on each topic. The first special issue of Electronic Markets on "Logistics" was published in March 1993 with a total of seven articles. Other special issues followed in 1999 on "Electronic Commerce and Logistics" with six articles as well as on "Electronic Markets and Supply Chains" with a total of four articles in 2010. Less coverage was dedicated to the area of academic publishing with one special issue on "Entertainment and Media" in 2002 with four papers, while another special issue on "Information systems research in the media industry" with three papers only followed in the last issue and another special issue on the transformation of the media industry that yet has to follow.

\section{Academic publishing industry}

As an academic journal, Electronic Markets is directly affected by the structural changes in academic publishing. Since becoming an academic journal, Electronic Markets has seen two major international publishers: Routledge (Taylor \&

Rainer Alt

rainer.alt@uni-leipzig.de

1 Information Systems Institute, University of Leipzig, Grimmaische Str. 12, 04109 Leipzig, Germany
Francis) between 1999 and 2009 and Springer since 2009 (Alt et al. 2015a). With the intent to grow their market share, both companies have pursued active acquisition strategies. For example, Taylor \& Francis merged with Informa in 2004 and purchased a number of smaller publishing houses, such as M.E. Sharpe in 2014. In the same vein, Springer acquired Palgrave Macmillan with important subsidiaries, such as the Nature Publishing Group, in 2015 and now figures as Springer Nature. These consolidations in the academic publishing market led to articles, such as "The 'Wild West' of Academic Publishing" (Lambert 2015), which report that subscription prices for academic journals "have risen at triple the rate of inflation for the past three decades [...] even though most now appear solely online, sparing their publishers the costs of printing and distribution". At the same time, many academic institutions have been facing budget restrictions, which prevents them from meeting the increased costs for academic publications - a situation that has been referred to as "the serials crisis” (Panitch and Michalak 2005, Kingsley 2006).

From many other industries, using information technology (IT) is known to have the potential to substantially reduce costs. For example, McKinsey reports broadly that "by digitizing information-intensive processes, costs can be cut by up to 90 percent" (Markovitch and Willmott 2014). The academic publishing value chain, which consists of the main steps investigation, creation, bundling and distribution (Alt and Militzer-Horstmann 2017), has seen many applications of IT. First of all, authors write their manuscripts directly in an electronic word processing system and have turned to access literature for their work via electronic instead of physical libraries. Large digital libraries are offered by academic publishing houses, such as ScienceDirect (Elsevier), SpringerLink (Springer Nature), and Tandfonline (Taylor \& Francis) as well as by consolidators, such as Ebsco and Proquest. In the last decade, the publishing houses have also implemented paper- 
handling systems that support the submission and review processes within the editorial offices (e.g. the Editorial Manager system by Aries Systems, which is also used by Electronic Markets). Together with paper publishing systems, the entire value chain in academic publishing is supported by IT, which - at least in principle - allows for an entirely electronic (or digital) value chain with the corresponding potentials in terms of speed and cost. However, much leeway for transformation still exists in this industry, especially, if compared with other information businesses, such as the music and the software industry.

\section{Cycle times in academic publishing}

Besides prices, which are regarded as mono- or oligopolistic and are still considered to yield comfortable profit margins for publishing houses, cycle times in academic publishing have only improved marginally with the technological innovations. Although authors have already been creating and delivering their manuscripts electronically, the subsequent bundling process - including the editing of content and layout - still requires several months for books as well as for journal publications. In the academic world, the peer-review system (Spier 2002) carries some imponderability in the bundling process, since the setting is more interorganizational or distributed in nature. Editorial offices are often located in an (academic) organization outside the publishing house, which again is different from the organization to which the voluntary reviewers belong. In fact, timeliness has repeatedly been recognized as a key shortcoming of the double-blind procedure (Ives 1993; Bailey et al. 2012). More specific research has been conducted on the issue of cycle times. For example, an in-depth analysis by Björk and Solomon (2013) summarized 14 past studies on the publication delay in academic publishing and revealed that the period from submission to publication varied between 2.5 and 36.5 months with an average of 15.26 months. The authors also conducted their own analysis of 135 journals from nine disciplines, which yielded an average time of 12.18 months. Three observations from these analyses are remarkable:

- First, the publication delay varies among academic disciplines. The shortest delays are observed in the natural sciences (in particular chemistry, engineering and biomedicine) while the longest cycles - with approximately 18 months almost twice as much as in chemistry - are present in the area of business and economics. This seems to be supported by the brief analysis of Ives (1993), who observes approximately 30 months for three IS-related journals.

- Second, the overall time was subdivided into the time for reviewing (i.e. submission to acceptance) and the time for production (i.e. from acceptance to publication). More or less both sub-phases had the same share within the overall process, i.e. the time for the review process may explain some delays in the publication process, but almost the same time elapses after the acceptance decision.

- Third, the journals that were created as open access (OA) had considerably shorter time-spans with 5.97 months overall and only 1.80 months for production. Although the authors refer to limitations due to a small sample size, research by Hartgerink (2015) confirms that production times have remained in this range (i.e. a mean of 50 days) for Public Library of Science (PLoS) journals even though review times have doubled up to 150 to 250 days on average.

These observations illustrate that even when sticking to the double-blind system, the immanent delays in the overall process are not time constants. Several scientific disciplines demonstrate short publication times, which suggests that higher efficiencies in the reviewing stage are still possible. An explanation may be the cultural differences in the respective disciplines, which lead participants, i.e. authors, reviewers and editors, to more or less adhere to their respective timelines. Everyone who has experienced the concurrency of commitments most researchers are typically facing knows that many sources for delivering late are possible (see Zmud 1997) and that short review cycles always depend on the collaboration of many parties. While delays in the review stage may be explained with academic tradition, the production stage is more technical in nature and multi-month production times are difficult to explain in the digital age. Could conceptual and technological advances known from other domains contribute to reducing the time problem within academic publishing? In fact, supply chain management (SCM) might provide some indications since it is not only linked to an industry, but also to some conceptual perspectives.

\section{Flow system perspective}

Originally, SCM has evolved in the 1980s from the logistics sector. Both, SCM and logistics, aim at designing systems of multiple actors, resources and goods, whereby SCM is more comprehensive. First, logistics is usually associated with an industry that focuses on organizing and operating flows of physical goods. Usually, this comprises storing and moving cargo from a sender to a recipient across multiple legs, i.e. air, rail, road and/or sea cargo. In addition to the interorganizational complexity, which may especially increase in international settings, these transport chains also have to meet requirements in terms of delivering goods at the right cost, quality and time. Advanced solutions are known in the area of courier, express and parcel services (CEP), which have 
provided time-definite overnight transportation globally since the 1980s. Companies, such as DHL or Fedex, were able to offer these services mainly since they owned most transportation resources along the chain and established electronic tracking systems to manage the chain early on (Moad 1998). The logistical principles to manage physical goods flow have given rise to another perspective on logistics, which is concerned with managing flow systems (Klaus 2009). Flow systems are not limited to physical parcels, but also occur when humans move in cities or organizations as well as when information is shared. In a nutshell, SCM adopts this thinking and not only focuses on physical goods flows, but also on flows of the associated information and financial goods (Lambert et al. 2005). Examples are order processing, inventory management, materials planning, and invoicing. Besides transportation, SCM considers the entire supply chain with the activities before and after transportation, such as purchasing and manufacturing. Thus, SCM applies a broader perspective and aims at managing the entire supply chain over multiple echelons, i.e. from the first supplier to the end-user (Naslund and Williamson 2010).

Flow systems are driven by customer demand and feature efficiency as well as flexibility. Demand signals are shared from the end of the chain to all stages involved, which allows to avoid buffers as well as inventories and to appropriately link the various (modularized) activities in the chain (Klaus 2009). Applied to the field of academic publishing this means that:

- Publications meet the customer's need. Obviously, this is not equivalent with adapting the research results themselves, but points at the efficient access of the desired content. For example, this could lead to distribution strategies that focus more on a topic-related preparation of content instead of having articles compiled in prearranged issues. We could also conceive modularized formats that make use of fragmented content, i.e. a personalized collection of findings from various articles instead of the entire article.

- Publications omit excess shelf inventory. While this requirement is self-explanatory for the replenishment of physical books or journals in stores and calls for just in time or on-demand publishing, most of today's academic literature is consumed electronically anyhow and thus practically avoids the inventory problem.

- Buffers need to be eliminated in the upstream process steps. In view of the cycle times mentioned above, this seems a critical inefficiency in existing peer-reviewing and publishing processes. Measures from SCM to improve the synchronization of activities via standardized interfaces, the introduction of process-wide control systems or process redesign (e.g. by adding, compressing, parallelizing, skipping or splitting activities) could be options for academic publishing as well (Fig. 1).

\section{Potentials for improvement}

As mentioned above, academic publishing processes have already received some streamlining in the past (e.g. Mandviwallaa et al. 2008). In a first phase this referred to using IT for mailing documents and decisions back and forth and in a second phase it comprised the implementation of electronic workflow systems for handling papers in the review process. Systems such as the Editorial Manager or Manuscript Central have improved the ease as well as the transparency of collaborating in these distributed settings by establishing a single point of information. Despite the fact that information from these systems may be obtained in real-time, this does not necessarily mean that this information is up-to-date and that processes are not delayed. For example, submissions may differ from the journal's formatting guidelines, contact details and the areas of interest of potential reviewers may be incomplete or outdated, reviewers may be declining invitations or not responding after having agreed, and authors may exceed their allowed time for revisions. Among the improvements that could contribute to increasing the publication flow are:

- Automation of routine checking. Next generation review systems could relieve editorial offices from manually checking whether the manuscript complies with the guidelines of the journal regarding de-personalization, the use of a given template and the completeness of all sections (e.g. abstract, personal details). This could build on existing formatting styles that are included for various journals in citation management tools, such as Endnote, Refworks, Mendeley or Zotero.

- Decision support for allocation tasks. The renaissance of artificial intelligence (AI) could add functionalities to review systems that address the allocation of reviewers to submissions. A recent contribution by Price and Flach (2017) conceived this decision as combinatorial allocation problem that may be solved via score matrixes based on available techniques from information retrieval and machine learning. The rationale is not only to reduce workload for responsible editors, but also to augment the probability that invited reviewers are more likely to agree if a paper falls within their area of expertise. At the same time, the hope is that, similar to the idea behind paper bidding processes at conferences this also positively influences the quality of reviews. As a further step, these could also be initially (pre-)rated by the system based on a knowledge base of previous reviews. Since the standards of evaluation differ among reviewers, in a system that has collected data on a researcher's reviewing history and has established reviewer profiles, the scorings could also take into account inter-reviewer differences. Although Price and Flach call for a global reviewing system, they are also aware of risks and other open issues that still need to be solved. 
Fig. 1 Value chain of academic publishing (adapted from (Alt and Militzer-Horstmann 2017) and (de Hon 2015))

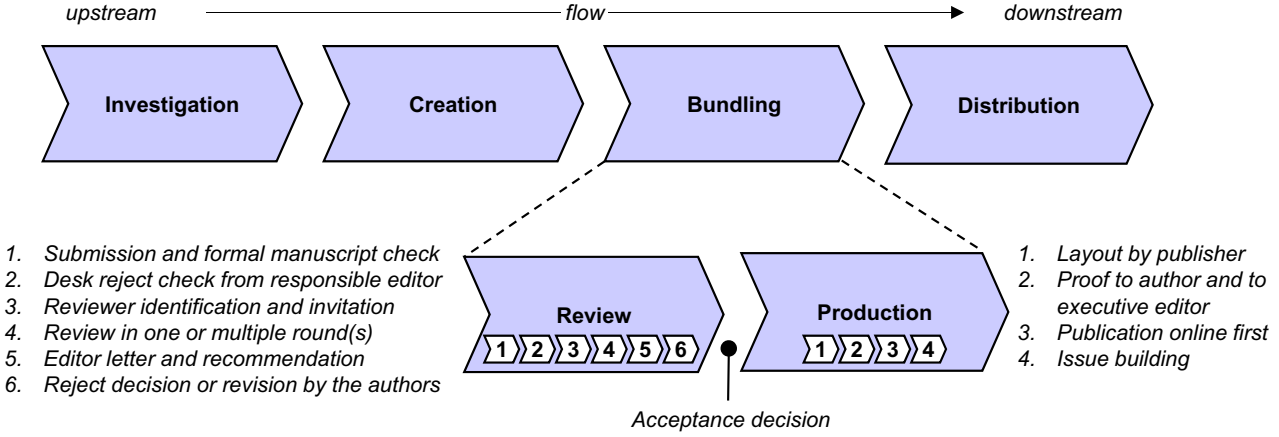

In summary, adopting a flow perspective from SCM could be helpful in unlocking potentials based on IT innovations, which are much needed in academic publishing. At the same time, each industry holds its specifics. For example, the products of academic publishing are different from industrial products, which are more deterministic in nature. Typically, each publication is a project and at least in the scientific world, professional and constructive reviews - whether open or closed - are expected to advance the results. While knowledge from SCM could contribute to improving the processes in academic publishing towards becoming less inefficient, identifying hints that could be obtained from academic publishing for the area of SCM appears more demanding. In the first place, the results obtained in scientific research may also prove relevant to practitioners in the SCM field as well. Second, if viewed as part of the media industry, the tradition of merging multiple media formats and data from various sources into new formats (Lugmayr 2017) could be helpful for supply chain researchers, e.g. in the context of risk management when information on exchange rates, strikes or other critical events is provided. Perhaps the present special issues also trigger ideas in this direction.

\section{Special issue, awards and mutations}

The first special issue is on "Electronic markets in supply chain management" and was organized by Günter Prockl from Copenhagen Business School, Denmark, Vikram Bhakoo from the University of Melbourne, Australia, and Christina Wong from the Hong Kong Polytechnic University. They introduce the three papers that were selected for the special issue more extensively in their preface "Supply Chains and Electronic Markets - Impulses for value cocreation across the disciplines" (Prockl et al. 2017). All three papers present different IT-driven perspectives on SCM, i.e. on interorganizational systems for maritime transport, on visibility services in various industries, and on Internet of Things applications. The guest editors team for the special issue on „Transformation of the academic publishing market" consisted of Diego Ponte from Trento University, Italy, Bozena Mierzejewska from Fordham 
University, USA, and Stefan Klein from the University of Münster, Germany. They introduce the three selected papers in their elaborate preface titled "The transformation of the academic publishing market: multiple perspectives on innovation" (Ponte et al. 2017) and provide valuable analyses for understanding the current situation, the digital transformation and the implications on the academic publishing industry. We are indebted to both teams for their effort and wish to thank all guest editors as well as the authors and reviewers for making these two remarkable special issues possible.

The second issue of each year also gives us the opportunity to present the results of the two annual Electronic Markets' awards. First, the "EM Outstanding Reviewer Award 2016" honors reviewers that repeatedly provided exceptional reviews in the past year. The nominees were determined from all reviewers that served in 2016 (see Alt and MilitzerHorstmann 2017) via a quantitative assessment of the reviewer ratings in the editorial manager system and a qualitative assessment of the respective reviews along the guidelines of Electronic Markets (see Alt et al. 2015b). While we appreciate the workload from all reviewers and editors, the three colleagues that convinced the editorial team the most with thorough, detailed and always constructive reviews in 2016 were Ulrike Lechner, Christian Matt and James E. Richard (see Table 1). Second, the "Paper of the Year Award 2016" aims to qualify papers that were outstanding among the articles published in Electronic Markets. Again, a two-step approach was applied to determine the nominees. In the first step, the publisher and the Electronic Markets' editorial office calculated the number of citations and downloads in 2016 for all articles published in Electronic Markets in 2015. This led to five papers, which were subsequently shared with the Senior and Associate Editors to obtain a voting on the top two papers taking into consideration the papers' fit to the journal's scope, its methodological rigor, and the contribution to theory as well as to practice. The two papers that succeeded are shown in Table 1 and were, remarkably, both published in the special issue on "Smart tourism: convergence of information technologies, experiences, and theories". We wish to congratulate all ten colleagues for their achievement!

Finally, Electronic Markets' Annual Editorial Board Meeting was held as ancillary event of ICIS 2016 in Dublin.
Besides announcing the organization of the Electronic Markets awards and an update on submissions and special issues, mutations in the board of Electronic Markets were discussed. First, two colleagues, who have accompanied Electronic Markets for many years have announced to end their term as Senior Editors of Electronic Markets. We are grateful to the service of Stefan Klein and Nicolas Romano, who together have handled more than 100 papers as associate and senior editors. Stefan has been a member of the editorial board since 1999 and became an Associate Editor in 2009 with a responsibility for quality management. Nicolas joined the editorial board in 2006 and became an Associate Editor responsible for presences at international conferences in 2009 as well. Both accepted to their role as Senior Editor in 2012 and convinced us and many authors with their feedback, which was always professional and thoughtful. We are highly indebted for the time, expertise and spirit they brought to Electronic Markets. Along with Stefan and Nicolas' departure as Senior Editors we would like to welcome two new Senior Editors, who have been elected by the editorial board: Steve Bellman, who has served as Associate Editor since January 2014, and Chris Holland, who has joined as Associate Editor in August 2013, have accepted this invitation. In addition, the board members also agreed to invite outstanding members of the editorial board as associate editors. Volker Bach from Steinbeis University Berlin, Germany, Ulrike Lechner from Universität der Bundeswehr in Munich, Germany, and Martin Smits from Tilburg School of Economics and Management, Netherlands have been members of Electronic Markets' editorial board for many years and have performed numerous reviews. In the same way, colleagues that have repeatedly provided profound and constructive feedback as reviewers were invited to the editorial board. We highly appreciate the readiness of the following five colleagues to strengthen the Electronic Markets board: Mathias Klier (Ulm University, Germany), Günter Prockl (Copenhagen Business School, Denmark), Susanne Leist (Regensburg University, Germany), Fábio Lobato (Federal University of Western Pará, Brazil) and Marijn Janssen (Delft University of Technology, Netherlands). We welcome all new senior and associate editors as well as board members and look forward to collaborating with all of them. In this process, a new name
Table 1 Electronic Markets awards 2016

\begin{tabular}{ll}
\hline EM outstanding reviewers 2016 & EM paper of the year 2016 \\
\hline - Ulrike E. Lechner, Universität der Bundeswehr, & $\begin{array}{c}\text { - Ulrike Gretzel, Marianna Sigala, Zheng Xiang and } \\
\text { Chulmo Koo, position paper "Smart tourism: }\end{array}$ \\
Munich, Germany & $\begin{array}{l}\text { foundations and developments" (Gretzel et al. 2015) } \\
\text { - Christian Matt, University of Bern, Switzerland } \\
\text { - James E. Richard, Victoria University of }\end{array}$ \\
$\begin{array}{l}\text { - Bellington, New Zealand } \\
\text { research paper "Smart technologies for personalized } \\
\text { experiences: a case study in the hospitality domain" } \\
\text { (Neuhofer et al. 2015) }\end{array}$
\end{tabular}


will appear in the context of the editorial team. Maxi Herzog has joined as Assistant Executive Editor in January and will support many day-to-day tasks. Welcome to her as well and with this we remain with

best regards from Leipzig and St. Gallen, Your EM-Team

\section{References}

Alt, R., \& Militzer-Horstmann, C. (2017). Electronic Markets on the media industry. Electronic Markets, 27(1), 1-5. doi:10.1007/s12525-017-0246-5.

Alt, R., Militzer-Horstmann, C., \& Zimmermann, H.-D. (2015a). Editorial 25/1: 25 years of electronic markets. Electronic Markets, 25(1), 1-5. doi:10.1007/s12525-015-0185-y.

Alt, R., Militzer-Horstmann, C., \& Zimmermann, H.-D. (2015b). Electronic Markets on reviewing. Electronic Markets, 25(4), 255261. doi:10.1007/s12525-015-0207-9.

Bailey, C. D., Hair Jr., J. F., Hermanson, D. R., \& Crittenden, V. L. (2012). Marketing academics' perceptions of the peer review process. Marketing Education Review, 22(3), 263-278.

Björk, B. C., \& Solomon, D. (2013). The publishing delay in scholarly peer-reviewed journals. Journal of Informetrics, 7(4), 914-923. doi: 10.1016/j.joi.2013.09.001.

De Hon, F. (2015). From submission to sharing: the life cycle of an article. November 2015, https:/www.elsevier.com/reviewers-update/story/ tutorials-and-resources/from-submission-to-sharing-the-life-cycleof-an-article.

Gretzel, U., Sigala, M., Xiang, Z., \& Koo, C. (2015). Smart tourism: foundations and developments. Electronic Markets, 25(3), 179188. doi:10.1007/s12525-015-0196-8.

Hartgerink, C. H. J. (2015). Publication cycle: a case study of the Public Library of Science (PLOS). https://www.authorea.com/users/2013/ articles/36067/_show_article. Accessed 13 March 2017.

Ives, B. (1993). Cycle-time reduction for disseminating scholarly research. MIS Quarterly, 21(3), xxi-xxiii.

Kingsley, D. (2006). Open access publishing: a solution to the serials crisis? Australasian Science, 27(2), 34-36.

Klaus, P. (2009). Logistics research: a 50 years' march of ideas. Logistics Research, 1(1), 53-65. doi:10.1007/s12159-008-0009-y.

Lambert, C. (2015). The "wild west" of academic publishing. Harvard Magazine, January-February 2015, pp. 56-83.
Lambert, D., García-Dastugue, S., \& Croxton, K. (2005). An evaluation of process-oriented supply chain management frameworks. Journal of Business Logistics, 26(1), 25-51. doi:10.1002/j.2158-1592.2005. tb00193.x.

Lugmayr, A. (2017). Information systems research in the media industry. Electronic Markets, 27(2), 7-8. doi:10.1007/s12525-016-0241-2.

Mandviwallaa, M., Patnayakunib, R., \& Schuff, D. (2008). Improving the peer review process with information technology. Decision Support Systems, 46(1), 29-40. doi:10.1016/j.dss.2008.04.005.

Markovitch, S., \& Willmott, P. (2014). Accelerating the digitization of business processes. Digital McKinsey, McKinsey\&Company, http:// www.mckinsey.com/business-functions/digital-mckinsey/ourinsights/accelerating-the-digitization-of-business-processes. Accessed 13 March 2017.

Matt, C., Hoerndlein, C., \& Hess, T. (2017). Let the crowd be my peers? How researchers assess the prospects of social peer review. Electronic Markets, 27(2). doi:10.1007/s12525-017-0247-4.

Moad, J. (1998). FedEx tracks customers on the move. PC Week, 15(48), 110.

Naslund, D., \& Williamson, S. (2010). What is management in supply chain management? - a critical review of definitions, frameworks and terminology. Journal of Management Policy and Practice, 11(4), 11-28.

Neuhofer, B., Buhalis, D., \& Ladkin, A. (2015). Smart technologies for personalized experiences: a case study in the hospitality domain. Electronic Markets, 25(3), 243-254. doi:10. 1007/s12525-015-0182-1.

Panitch, J.M. \& Michalak, S. (2005). The serials crisis. A white paper for the UNC-Chapel Hill scholarly communications convocation, January, 2005.

Ponte, D., Mierzejewska, B., \& Klein, S. (2017). The transformation of the academic publishing market: multiple perspectives on innovation. Electronic Markets, 27(2). doi:10.1007/s12525-017-0250-9.

Price, S., \& Flach, P. A. (2017). Computational support for academic peer review: a perspective from artificial intelligence. Communications of the ACM, 60(3), 70-79. doi:10.1145/2979672.

Prockl, G., Bhakoo, V., \& Wong, C. (2017). Supply chains and electronic markets - impulses for value co-creation across the disciplines. Electronic Markets, 27(2). doi:10.1007/s12525-017-0253-6.

Spier, R. (2002). The history of the peer-review process. Trends in Biotechnology, 20(8), 357-358. doi:10.1016/S0167-7799(02) 01985-6.

West, J. D., Bergstrom, T., \& Bergstrom, C. T. (2014). Cost effectiveness of open access publications. Economic Inquiry, 52(4), 1315-1321. doi:10.1111/ecin.12117.

Zmud, B. (1997). Reducing the cycle-time of the MIS Quarterly's review process. MIS Quarterly, 21(3), xxxv-xxxix. 